\title{
Direct selection by color for visual encoding
}

\author{
ESTHER VIERCK and JEFF MILLER \\ University of Otago, Dunedin, New Zealand
}

\begin{abstract}
In two experiments, we used rapid serial visual presentation tasks to examine the usefulness of color for the direct selection of visual information for perceptual encoding. The participants' task was to make a discrimination as to whether a target letter within a rapid sequence appeared in its upper- or lowercase version, and an advance cue indicated the color in which the target letter was most likely to occur. To maximize the usefulness of the cued color, in validly cued trials, we used sequences in which the target was the only item appearing in the cued color. In both experiments, accuracy was highest for validly cued trials. A cost-benefit analysis revealed a facilitory effect of valid cues and an inhibitory effect of invalid ones. These results support the idea that color cuing allows the direct selection of objects for further perceptual processing.
\end{abstract}

When we look for a target object in a visual scene, we often need to be able to single out or select that object so that it can be processed further once it is found. One interesting question regarding this selection process is to consider what types of information can guide it. Within research on selective attention, a common method for examining this question is to provide a cue giving an observer advance knowledge about some aspect of the target object or the setting in which it will appear. If the cue aids selection, then the information it provided must have been useful in guiding selection.

Researchers have examined several types of cuing information to determine their potential benefits for selection, and the results clearly indicate that spatial information is the most useful. In one study, for example, Posner, Snyder, and Davidson (1980) asked their participants to respond rapidly to the onset of any one of four light-emitting diodes. Prior to stimulus onset, the participants were given a cue indicating the likely location of the target diode onset. Responses were faster when the cue was correct (valid cue trials) than when it was incorrect (invalid cue trials). Thus, a location cue allows participants to attend selectively to the indicated location, so a target can be detected better at that location than at uncued locations. Similar results have been reported in paradigms in which identification tasks (Cheal \& Gregory, 1997) and discrimination tasks (Theeuwes, 1989), rather than simple detection tasks, such as that in Posner et al., have been used. In short, a variety of results have provided evidence that location cues offer significant benefits for selection.

Color is another feature that has been examined for its capacity to aid selection. The results of studies in which

This research was supported in part by a Marsden grant from the Royal Society of New Zealand to J.M. We thank Y. Tsal and an anonymous reviewer for helpful comments on an earlier version of the manuscript. Correspondence concerning this article should be addressed to E. Vierck or J. Miller, Department of Psychology, University of Otago, Dunedin, New Zealand (e-mail: esther@psy.otago.ac.nz or miller@psy.otago.ac.nz). target color was cued have indicated that advance color cues generally provide some selection advantage, although not as much as location cues. For example, Carter (1982) presented search displays consisting of multiple three-digit numbers. Participants had to find the threedigit number beginning with two specified target digits and then report the third digit within that number. Responses were faster when the color of this number was cued than when it was not. Carter also manipulated the number of items with the cued color while keeping the total display size constant, and he found that search times varied according to the number of items in the cued color but were largely independent of the number of items in the other colors. Similar results have been reported for tasks in which letter discrimination (LaBerge \& Brownston, 1974 ) and search for two-digit numbers (Green \& Anderson, 1956) were used. Thus, these results clearly demonstrate that color cues can aid selection by defining a subset of items for selective processing. ${ }^{1}$

\section{Is Selection by Color Completely Mediated by Location?}

Although it is quite clear that color information can be used to guide selection, it is less clear how it does so. At one extreme is the possibility that the advantage for items in a cued color is mediated entirely by location. According to this view, color cues enable observers to direct attention to locations at which the cued color is present, thereby allowing further processing of the items at these locations. On this view, there is no advantage for an item of the cued color beyond the increased probability that its location will be selected. At the other extreme, however, is the possibility that items in the cued color can be selected directly, leading to directly enhanced processing - that is, processing not mediated by location-of items appearing in the cued color. Many studies have provided data bearing on this distinction, but none has settled it decisively. In particular, the available data indicate that at least part of the advantage conveyed by color cuing is 
mediated by location, but they do not clearly indicate whether all of the advantage is. The experiments reported in this article were conducted in an attempt to provide a stronger test for location-independent effects of color cuing. To set the stage for these experiments, we first will review previous studies concerning location-dependent and location-independent effects of color cues.

Brawn and Snowden (1999) provided evidence that at least some of the advantage for cued colors comes from the direction of attention to the cued locations. They presented an array of elements, one of which would change luminance after a variable amount of time. Participants were required to discriminate whether a decrease or an increase of luminance took place. Half of the elements were red, and the other half were green. Before some trials, an informative cue was presented indicating the color of the element that would change luminance in that trial. Responses in these trials were significantly faster than those with uninformative cues. In one experiment, they also manipulated the intensity of the luminance change. They argued that if all of the effect of color cuing occurred after attention had been directed to the item's location, the cuing effect should be the same for weak versus strong flashes. They found, however, that the cuing effect depended on flash intensity, so they concluded that color cuing had its effect before attention reached the target location - that is, that color was used to select the appropriate location. This leaves open, however, the question of whether color is used only to select an appropriate location or whether color can have additional effects on selection other than that.

\section{Evidence in Favor of Complete Mediation by Location}

The hypothesis that color selection is completely mediated by location has been supported by several studies in which the mechanisms underlying the effects of color cuing have been examined. For example, Moore and Egeth (1998) had participants detect a digit in a display consisting of letters. Targets and distractors were either green or blue, and the participants were informed about the probability of the target being in one of these colors. The higher the probability for a specific color, the faster were the responses to targets in that color, suggesting that selection by color was effective. In a further experiment, however, the display was presented briefly and masked, and color cuing was not effective in raising the probability of correct target identification. Moore and Egeth concluded, then, that direct selection by color was not effective and that, instead, the participants simply selected items in locations with cued colors. It was still necessary to shift attention to those items, however, so cuing was ineffective if masking removed the display before the shift could be completed. That is, these results were interpreted to mean that the only function of color was to guide attention to specific locations.

The results of several other experimental paradigms have also been interpreted as indirectly supporting the idea that location fully mediates color-cuing effects. For example, Tsal and Lavie (1993) used a design with two possible target positions to the left and right of fixation. Color cues were presented on either the left or the right side, and participants were instructed to report either the right or the left letter, depending on the color of the cue, with the cue's position being irrelevant. The participants reported the correct letter more rapidly when it was adjacent to the location of the cue than when it was in the opposite position, indicating that the participants were not able to ignore the location of the cue. These results were interpreted as evidence that location has a special status in selection. Although this notion is not undisputed (Gold \& Pratt, 2001; van der Heijden, Kurvink, de Lange, de Leeuw, \& van der Geest, 1996), the special status of location is supported by many results from other paradigms (Cave \& Pashler, 1995; Garner, 1987; Kim \& Cave, 2001; Lamy \& Tsal, 2000; Nissen, 1985; Shih \& Sperling, 1996; Tsal \& Lamy, 2000; Tsal \& Lavie, 1988). The importance of location demonstrated in these studies, however, does not eliminate the possibility that color has some effect other than that mediated by the more effective cue of location.

\section{Evidence Against Complete Mediation by Location}

Studies in which both location and color were examined as selection cues have provided some evidence of an influence of color beyond pure guidance to location. For example, Lambert and Corban (1992) cued both the most likely location and the most likely color of the target stimulus in an orientation discrimination task. In every trial, participants were informed about the likely color of the target stimulus that was to follow. This color information also cued a likely location in the display; for example, a green cue was most likely followed by a green stimulus in the upper of two possible locations. In invalid trials, color expectations, location expectations, or both could be violated. Performance was better for stimuli displayed in the cued than in the uncued color, but the major influence on performance was the validity of the location expectation, indicating a dominant influence of location information. More important, in trials in which the color information was invalidly cued, responses were faster when the location of the displayed stimulus was associated with that of the actually displayed color than when it was associated with the cued color. Thus, performance was facilitated by learned combinations of color and location beyond what could be attributed to location alone, suggesting that the effect of color is not mediated completely by location.

In a different paradigm, Humphreys (1981) also found an advantage of combined color-location expectations over location information alone, analogous to that demonstrated by Lambert and Corban (1992). Humphreys had participants judge the orientation of a form that always appeared at the same location. A distractor form was presented next to this target form in either the same or a dif- 
ferent orientation. Humphreys compared reaction time (RT) and accuracy in one condition in which the orientation of the distractor form changed randomly from trial to trial versus another condition in which the orientation of the distractor form was constant across a whole block of trials. When the target and the distractor were displayed in the same color, performance was better in the constant condition than in the random condition. If the target was displayed in a different color than was the distractor, however, RT and accuracy were similar in the two conditions. Thus, in this task, prior knowledge of location did not eliminate the influence of the incompatible distractor form, but knowledge of location plus color made the selection successful. The author concluded that attention can be allocated to either color or location, depending on task demands.

The results of Lambert and Corban (1992) and Humphreys (1981) suggest that color cuing may have effects in addition to location cuing, and they thus provide some support for the hypothesis of direct selection by color. There is, however, another interpretation of the results that is consistent with the hypothesis that all color-cuing effects are mediated by location: Even when location cues are given, color might still be used as an additional cue for selecting the appropriate location, especially when display locations are nearby and confusable in the first place, as they were in these studies. That is, color might actually just have provided further help to lead attention to the appropriate location in these studies. In that case, the effects of color cues in these studies would have been mediated by location after all.

\section{Studies Testing for Direct Selection by Color Without Variation in Location}

As the above review highlights, it is very difficult to be sure from studies with different colored items in different locations whether benefits associated with color cuing happen directly or are mediated by location. In order to isolate a direct selective capacity of the visual system for color, it is necessary to disentangle the confounding of location and color that is present in most displays with multiple colored items. This has been done in a number of paradigms, but the results are mixed regarding the issue of whether direct selection by color is possible.

One way to disentangle color and location is to present overlapping stimuli in different colors and instruct participants to pay attention to a stimulus of a particular color. Studies in which this approach has been used have generally shown evidence that participants can select by color. For example, Rock and Gutman (1981) presented participants with two superimposed shapes of different colors. The task was to give a subjective judgment about the shape of one color and to ignore the shape of the other color. Later, the participants were given a surprise recognition task for all of the shapes, and recognition was substantially better for shapes that had been presented in the attended color than for those presented in the unattended color. Similarly, Goldstein and Fink (1981) used superimposed colored line drawings of scenes, instead of novel shapes, and they obtained comparable results. Furthermore, in a study of negative priming, Tipper and Cranston (1985) found that participants could consistently report the identity of one of two superimposed items presented in a prespecified target color. These findings clearly demonstrate that color can be used as the defining feature for selection processes, and the fact that the attended and the unattended stimuli were superimposed suggests that mediation by location was unlikely. Nonetheless, Brawn and Snowden (1999) have argued that selection with overlapping stimuli might still have been based on spatial or object-based properties, rather than on color per se.

Other studies have shown that participants can reliably pick out a target stimulus defined by color when it is presented in a rapid sequence of superimposed stimuli (rapid serial visual presentation, or RSVP, task; Botella \& Eriksen, 1992; Chun, 1997; Folk, Leber, \& Egeth, 2002; McLean, Broadbent, \& Broadbent, 1983). Clearly, in these paradigms, the participants were able to select objects for report on the basis of color. Furthermore, with a rapid sequence of stimuli in the same location, it seems impossible that selection was mediated by location.

Although the experiments with overlapping stimuli and the RSVP paradigm have demonstrated a direct selective capacity for color, an important limitation of these studies for the present purposes is that it is not clear at what processing level this selection took place (cf. Brawn \& Snowden, 1999). In particular, in these paradigms selection might have taken place rather late, after full attended perceptual processing of superimposed stimuli. In both Rock and Gutman's (1981) and Goldstein and Fink's (1981) studies, for example, the selective effect was observed in recognition memory performance, so attentional selection by color might have influenced only the rehearsal of items, not their original encoding. Similarly, in Tipper and Cranston's (1985) experiment, selection for report might have taken place after perceptual encoding of both items was complete. Indeed, the negative priming effect observed by Tipper and Cranston provides some evidence that the stimulus in the unattended color was encoded to some extent. Finally, in the RSVP tasks, it is possible that all the items in the RSVP sequence were encoded equivalently and, afterward, only the item of the cued color was selected for report or rehearsal.

In two studies, multielement RSVP tasks have been used in more elaborate attempts to isolate direct selection by a cued color. Shih and Sperling (1996) conducted an experiment in which each RSVP frame contained six items. Participants searched for the one digit among letters and reported its location within the frame, its identity, and its color. The authors compared performance in three conditions: (1) Frames were displayed in alternating colors, and the target color was cued; (2) every second frame was blank; and (3) all the frames were in the same color. If selection by color was perfect, the number 
of frames would be halved by the informative color cue, so performance in that condition would be equivalent to performance in the condition with blank frames. If selection by color was not at all helpful, however, performance in the alternating-color condition should be similar to performance in the same-color condition. The results showed an advantage for the blank condition and no difference between the alternating-color condition and the same-color condition, suggesting that selection by color was not at all helpful and arguing against the hypothesis of direct selection by color.

Although Shih and Sperling's (1996) results seem to provide strong evidence against the possibility of direct selection by color, several aspects of this study cast doubt on this conclusion. One important aspect lies in the effectiveness of the cue in the alternating-color condition. Even in the $100 \%$ valid cue condition, the cue would have led only to a $50 \%$ reduction in the number of relevant frames - that is, it would still have left 10-13 relevant frames, depending on sequence length. This is in contrast to typical location-cuing experiments (e.g., Posner et al., 1980; Treisman, 1985), in which a valid cue leads to a reduction from several possible locations to a single location. The color cue used by Shih and Sperling was thus much less informative than the location cues used in previous location-cuing experiments, and therefore, the participants might have been less inclined to make use of it. Another potentially important aspect of the paradigm concerns the processing demands in the alternating-color condition. In that condition, the participants had to switch constantly between two different search procedures: They first had to select in the time domain by color, and then, if the color was correct, they had to select in the spatial domain by category. Such complicated selection procedures might interfere with the use of color cues. A final, potentially problematic aspect of the design is that the validity of the color cueranging from $20 \%$ to $100 \%$-was specified independently for each trial. It might have been that the switches between different cue validities were too capacity demanding, especially within a paradigm that was rather complicated in the first place. Since spatial search for the target location was necessary to find the target anyway, the participants might have restricted their efforts to the spatial search and not made use of the color cues.

A similar experiment was carried out by Põder (2001). He avoided the confounding factor of spatial influence within frames by using an RSVP paradigm with a single stimulus per frame to study the possibility of color-based selection. Like Shih and Sperling (1996), Põder used alternating colors for his frames. In addition to prior information about the target form, he also provided color cues, thereby enabling the participants to reduce the total number of frames by half. In half of the trials, valid color cues were presented. This color information did not lead to better performance than a no-color-cue condition did, however, and Põder concluded that color was not useful in selection. But as in Shih and Sperling's study, a po- tential problem with the study is that the color cue had only limited usefulness, because many distractors appeared in the cued color, as well as the target. Again, the small potential benefit might not have been enough to motivate the participants to make use of the cue. Thus, although these two studies did not demonstrate any direct selection by color, it is still possible that such selection could be demonstrated in a design giving participants more incentive to use color information.

In summary, there is a great deal of evidence concerning people's ability to select a stimulus on the basis of its color, but it is still not clear whether this selection is always mediated by location or not. The most diagnostic studies appear to be those in which location and color are unconfounded by the use of overlapping stimuli, possibly embedded in an RSVP task, but the results of these studies do not agree. Moreover, it seems clear that each of the studies can be questioned on the basis of its particular features. When evidence suggesting that direct color selection is possible has been found (e.g., Rock \& Gutman, 1981), the selection might have taken place after perceptual identification of all the stimuli. When evidence suggesting that direct color selection is not possible has been found (e.g., Shih \& Sperling, 1996), it is possible that the conditions were not optimal for color selection in the first place.

The present experiments were further attempts to see whether color could be used for direct, early selection of visual information for perceptual encoding without mediation by location. Because of the difficulties inherent in interpreting results from experimental designs with stimuli varying in location as well as in color, we chose to use an RSVP paradigm with color cues. We also used a single stimulus per frame, to make sure that cuing effects were not mediated by location and to minimize the influence on selection of the possibly dominant factor of location. In contrast to the two previous studies (Põder, 2001; Shih \& Sperling, 1996) with this paradigm, however, we used color cues in a manner more similar to the way in which location cues have been used in the past. Specifically, in our designs, the information provided by the color cue isolated a single frame in which the target stimulus was most likely to occur.

\section{EXPERIMENT 1}

In the first experiment, participants performed a letter discrimination task within an RSVP paradigm. In each trial, the participants were required to search for a specified target letter and to indicate whether this letter appeared in uppercase or lowercase. The most likely color of the target was cued, and then an RSVP sequence of 15 colored letters was presented. Note that, as in the experiments of Brawn and Snowden (1999), Humphreys (1981), Põder (2001), and Posner et al. (1980), the cued information was not the defining feature of the target. Instead, it represented additional information and was not necessary to fulfill the task, because the participants could 
focus on the shape of the target letter alone to make a correct decision. This is similar to previous designs in which the selective qualities of location were revealed by an advantage for performance in valid cue trials (e.g., Posner et al., 1980; Treisman, 1985).

The target appeared in the cued color in $80 \%$ of all the trials. We refer to these as valid cue trials, because the information provided led to the correct target letter. For the remaining $20 \%$ of the trials, the target appeared in some other color, and the cued color did not appear anywhere in the sequence. We will refer to this type of cue as a neutral cue, because it conveyed no information that could be used to find the target within that particular RSVP stream. If items in a cued color can be directly selected for encoding, participants should be able to respond more accurately when cuing is valid than when it is not. Note that in the neutral trials, the cue was not actually misleading, as the term invalid implies. A more misleading invalid condition was introduced in Experiment 2.

\section{Method}

Participants. The participants were 32 undergraduate psychology students (5 males) at the University of Otago, New Zealand. Their ages ranged from 18 to 27 years. Their participation was part of a 1 st year course requirement. All the participants reported having normal color vision.

Stimuli. The stimuli were the upper- and lowercase versions of the letters F, H, R, and T, displayed in the triplex font provided by Turbo Pascal (Version 7). A color set of six different colors was used; these were blue (RGB 5,14,63), yellow (RGB 63,63,0), red (RGB 63,9,1), green (RGB 5,63,8), gray (RGB 16,16,16), and white (RGB 63,63,63). The lowercase form of the letter $r$ had a height of $8 \mathrm{~mm}$, and all other letters had a height of $12 \mathrm{~mm}$. The widths of the letters varied from 5 to $9 \mathrm{~mm}$. The cue consisted of 14 colored horizontal lines, each 1 pixel wide. Together, these lines created the image of a rectangle with a height of $14 \mathrm{~mm}$ and a width of $23 \mathrm{~mm}$. The stimuli were presented on an IBM-PC-compatible computer and were viewed from a distance of approximately $52 \mathrm{~cm}$.

All the stimuli were presented one at a time in the center of the screen on a dark background. The participants were required to respond with their index fingers. The index finger of the right hand pressed the slash (/) key, and the index finger of the left hand pressed the $\mathrm{z}$ key on a standard computer keyboard to indicate the case version of the target letter. The assignment of the response keys to upper- or lowercase letters was counterbalanced across participants.

Procedure. Experimental testing was conducted in a small semidarkened experimental chamber with a desk lamp as the only light source other than the computer monitor. Each participant was tested in eight blocks of 54 trials in a single session lasting approximately $45 \mathrm{~min}$. The first 4 trials in each block were considered warmup and were not recorded.

At the beginning of each block, the target letter for the block was displayed for $500 \mathrm{msec}$ in white, with a height of $30 \mathrm{~mm}$ and a width of $23 \mathrm{~mm}$. A different target letter out of the letter set was randomly chosen for every new block, subject to the restriction that consecutive blocks always had different target letters.

Each trial started with a plus sign, which served as a warning signal and a fixation point for the participant. The plus sign was presented for $800 \mathrm{msec}$ and was followed immediately by a colored cue in the form of a rectangle. The color of the cue was chosen randomly out of the color set. The cue was presented for $1,000 \mathrm{msec}$. The RSVP sequence began $200 \mathrm{msec}$ after the offset of the cue, and it consisted of 15 letters. Each letter was presented for $70 \mathrm{msec}$ and was immediately followed by the next letter. One of the letters was the target letter in either its uppercase or its lowercase form. Both ver- sions were equally likely and randomly chosen. The target appeared equally often and in a random fashion in the following position ranges within the 15 -item sequence: $1-3,4-6,7-9,10-12$, and 13-15. Within these categories, the position was chosen randomly.

In $80 \%$ of the trials, the cue was valid, so the color of the target letter was the same as the color of the cue. The other letters of the sequence consisted of a random mixture of upper- and lowercase versions of the other letters in the letter set, displayed in the five remaining colors of the color set. The cue color was used only for the target letter and did not appear elsewhere in the sequence. In the other $20 \%$ of the trials, the cue was neutral, so the target letter was of a color different from that of the cue. In neutral trials, the cued color was not at all used in the sequence.

A trial ended with the presentation of a question mark signaling the end of the trial. The question mark stayed on the screen until a response key was pressed or 5,000 msec had passed. Visual feedback was provided for correct and incorrect responses. In cases of errors in operation - for example, using the wrong response keys or responding before the target was shown - a visual reminder of the instructions was displayed on the screen. The next trial began after approximately $800 \mathrm{msec}$.

The participants were instructed to respond immediately after the detection of the target letter and not to wait for the question mark. They were also informed that the target's color usually was that of the cue, which was therefore potentially helpful, and that they were free to use this information to help them accomplish the task.

\section{Results and Discussion}

Only trials with RTs longer than $200 \mathrm{msec}$ and shorter than 2,500 msec were included in the analysis of RT. Five percent of the trials did not fulfill these criteria; of these, the vast majority were early responses. For the responses within the range of $0-200 \mathrm{msec}$, accuracy was at chance level. These early responses varied with target location, in that they became more frequent the later in the sequence the target was presented, suggesting anticipation. For the analysis of accuracy, all the trials were included, because limits would eliminate responses to letters presented before the target letter or responses in trials in which the participant missed the target and had to guess after the presentation of the last frame. All statistical tests were conducted using analyses of variance (ANOVAs), and Greenhouse-Geisser corrections (Huynh, 1978) were performed and are reported where appropriate.

Mean RT and percentage correct were calculated and averaged across participants, and the average RTs and accuracies for the valid and neutral color cue conditions are displayed in Table 1. On average, performance was better with valid cues than with neutral ones for both dependent measures.

ANOVAs were used to evaluate the statistical reliability of the within-subjects effect of cue (valid vs. neutral). For accuracy, the $6.4 \%$ advantage for the valid condition

Table 1

Average Percentage Correct (PC) and Reaction Time (RT, in Milliseconds) as a Function of Cue Validity in Experiment 1

\begin{tabular}{ccc}
\hline \multirow{2}{*}{ Trial } & \multicolumn{2}{c}{ Dependent Variable } \\
\cline { 2 - 3 } Condition & PC & RT \\
\hline Valid & 78.2 & 750 \\
Neutral & 71.8 & 899 \\
\hline
\end{tabular}


was highly significant $[F(1,30)=29.86, p<.001]$, as was the 149 -msec difference for RT $[F(1,30)=52.51$, $p<.001]$.

The only other significant result in the analysis of the accuracy data involving validity was the interaction between target case and validity $[F(1,30)=8.46, p<.01]$. Responses tended to be more accurate for lowercase targets than for uppercase targets, but this difference was much larger for neutral trials $(7.8 \%)$ than for valid trials $(0.3 \%)$. It appears that this interaction arose at least partly because of a bias toward lowercase responses. Uppercase responses were given in $46 \%$ of all the neutral trials, whereas lowercase responses were made in $54 \%$ of all such trials. It appears from the RT data presented next that uppercase targets were easier to detect than lowercase targets. As a result, the participants might have felt that they were more likely to miss lowercase targets, and therefore, they might have tended to decide in favor of a lowercase response whenever they had to guess. Because the additional color information was missing in the neutral trials, guesses would have been more common in this condition, leading to the interaction. Target case also interacted with target position and group $[F(4,120)=2.65$, $p<.05]$, apparently because the participants not only had a bias toward lowercase targets but also tended to use their dominant hand more often when they had to guess. The only other significant effect in the analysis of accuracy was that of target position $[F(4,120)=2.92, p<$ $.05]$, with responses becoming less accurate the later the target was presented in the sequence.

In the analysis of RT, there were several significant effects, in addition to that of validity. There was an effect of target position on RT, with faster responses to targets appearing later in the sequence $[F(4,120)=179.62, p<$ $.001]$. Together with the observed decrease in accuracy over target position, this suggests the presence of a speedaccuracy tradeoff. In addition, the effect on RT might reflect heightened motor preparation with an increase of target probability over the time course of the RSVP sequence (Miller \& Schröter, 2002). Not surprisingly, target position interacted with validity for RT $[F(4,120)=$ $15.42, p<.001]$, reflecting a decrease of the difference between valid and neutral trials, with faster responses in the later target positions. RT was also affected by practice, tending to decrease across blocks $[F(1,30)=8.55$, $p<.01]$. RT also differed for the upper- and the lowercase versions of the target $[F(1,30)=26.43, p<.001]$, with faster responses for the uppercase targets. This effect of case on RT decreased at later target positions $[F(4,120)=6.14, p<.001]$, consistent with the overall speedup of responses at later target positions.

The first experiment showed strong validity effects associated with color cues. When the color information given by the cue was valid, performance advantages could be observed in both measurements, accuracy and RT. These advantages suggest that color can be used to directly select items out of an RSVP sequence for the further processing needed for the discrimination be- tween upper- and lowercase. Because spatial location was kept constant, location could not have mediated selection. Moreover, it seems clear that selection must have preceded full stimulus identification, because of the task requirements. If all the stimuli were identified fully before color selection, the participants should have been able to select the target and report its case simply on the basis of its letter identity. That is, although focusing on the target form alone was logically sufficient to perform the task, the participants nonetheless benefited from advance knowledge of the target color. This effect of color cuing is in many ways analogous to the effect of location cuing shown by Posner et al. (1980), who found benefits for location cues despite the fact that the information conveyed by the cue was not necessary to do the task.

\section{EXPERIMENT 2}

In the second experiment, we sought to gain more information about the processes involved in selection by color by comparing facilitory and inhibitory effects of the color cues. For that purpose, we added a misleading invalid condition in which a distractor item appeared in the cued color. In these trials, selection of the cued color would lead to processing of an incorrect letter, presumably taking resources away from perceptual processing of the actual target letter and interfering with performance. Thus, a comparison of the neutral condition with the invalid condition allowed us to assess the costs associated with an incorrect color cue. As in Experiment 1, the strength of facilitation due to cuing can be judged by comparing performance in valid trials with that in neutral trials. These comparisons differ somewhat from those used in most previous cuing studies, in which costs and benefits have been assessed relative to a neutral condition in which no cues were given (e.g., Posner et al., 1980). As has been discussed by Jonides and Mack (1984), however, such comparisons make strong and rather doubtful assumptions about how participants perform in the absence of a cue. Thus, we preferred to compare only cued conditions and, therefore, chose a manipulation of validity that involved changes only in the RSVP sequence.

A secondary purpose of this experiment was to gain more information about how color is used by the selection process. Given the results of the first experiment, suggesting that color is a useful selector in a discrimination task, there are at least two possible accounts for this selection. One possibility is that items in the cued color were selected and assigned higher priority prior to shape processing. Another possibility, however, is that the participants created two mental images or templates for which they searched - that is, upper- and lowercase target letters in the cued color. These two possibilities cannot clearly be distinguished on the basis of the results of the first experiment, but the present experiment did allow us to separate them. In invalid trials, the cued color would lead the participants to select a letter that was not the target. This letter would have to be fully identified before 
it could be rejected. Thus, if the participants initially selected items on the basis of color and then processed the shape, they should sometimes respond on the basis of the nontarget letter in the cued color. On the other hand, if they searched only for a template containing a conjunction of features - namely, a target in the cued color - they should not respond to nontargets in the cued color, because these items do not satisfy both the criteria defining the template. An analysis of the error responses in invalid trials allowed us to distinguish these two possibilities.

\section{Method}

Participants. Twenty-four undergraduates (13 males) at the University of Otago, New Zealand, participated in this experiment. Their ages ranged from 17 to 42 years. They were reimbursed for their participation with NZ\$ 7. None of the participants was color-blind.

Stimuli and Procedure. All aspects of the stimuli and the procedure were the same as those in Experiment 1, except that a third condition was included. The valid and neutral conditions were the same as those in Experiment 1, and the new condition was an invalid condition in which one nontarget letter was randomly selected to be presented in the target color, whereas the target was presented in some other color. Each block consisted of 60 trials, 40 valid, 10 neutral, and 10 invalid, intermixed in random order.

\section{Results and Discussion}

For the analyses of response latencies, only trials with RTs longer than $200 \mathrm{msec}$ and shorter than 2,500 msec were included. Of the trials, $4.29 \%$ did not fulfill these criteria. Again, the vast majority of these trials were due to early responses, and responses with RTs between 0 and $200 \mathrm{msec}$ were at chance accuracy. As in the first experiment, early responses varied with target location, suggesting anticipation. Again, all the trials were included in the analysis of accuracy.

Average RTs and accuracy levels as a function of validity condition are displayed in Table 2 . The effect of validity was highly significant for both accuracy $[F(2,44)=16.36$, $p<.001]$ and $\mathrm{RT}[F(2,44)=27.05, p<.001]$. Pairwise comparisons between the different validity conditions, of course, showed highly significant advantages for valid over invalid trials of $10.0 \%$ for accuracy $[F(1,22)=25.89, p<$ $.001]$ and of $74 \mathrm{msec}$ for $\mathrm{RT}[F(1,22)=35.91, p<001]$. More important, the benefit of the valid condition, in comparison with the neutral condition, was also highly significant, amounting to $7.5 \%$ for accuracy $[F(1,22)=$ $11.73, p<.005]$ and to $127 \mathrm{msec}$ for $\mathrm{RT}[F(1,22)=$ $31.48, p<.001]$. Responses in neutral trials were only $2.5 \%$ more accurate than those in invalid trials, but this difference also reached significance $[F(1,22)=4.87$, $p<.05]$. Unexpectedly, analyses of RT showed that re-

Table 2

Average Percentage Correct (PC) and Reaction Time (RT, in Milliseconds) as a Function of Cue Validity in Experiment 2

\begin{tabular}{ccc}
\hline \multirow{2}{*}{$\begin{array}{c}\text { Trial } \\
\text { Condition }\end{array}$} & \multicolumn{2}{c}{ Dependent Variable } \\
\cline { 2 - 3 } & PC & RT \\
\hline Valid & 77.2 & 860 \\
Neutral & 69.7 & 987 \\
Invalid & 67.2 & 934 \\
\hline
\end{tabular}

sponses were $53 \mathrm{msec}$ faster in the invalid condition than in the neutral condition $[F(1,22)=11.75, p<.005]$. An examination of the RT frequency distributions showed that this difference was due mainly to a higher frequency of rather slow responses $(>1,400 \mathrm{msec})$ in neutral trials. It might be that in these trials, the participants were waiting for the cued color to appear and did not realize that they had missed the target letter until the sequence ended. RTs in the range of 200-400 msec from the presentation of the target frame were also more frequent in invalid trials than in neutral trials. This difference was caused mainly by errors in the invalid condition, reflecting responses to the nontarget in the cued color. Together, these explanations can account for the faster RTs in invalid trials.

The position of the target within the sequence only affected RT, with faster responses to targets later in the sequence $[F(4,88)=118.41, p<.001]$. As in Experiment 1 , the analysis of RT yielded a significant interaction of target position with validity $[F(8,176)=4.22, p<.005]$, reflecting a reduction of the difference between the three validity conditions at later target positions, presumably due to the overall decrease in RT at these positions. Similarly, there was a practice effect over blocks for RT $[F(1,22)=22.70, p<.001]$, but not for the accuracy data.

Although the pairwise comparison between the neutral and the invalid trials reached significance, this comparison might not be the most sensitive analysis with which to determine costs in this experiment. Consider an invalid trial in which the nontarget in the cued color appears later in the sequence than does the target. In such a trial, the system is in exactly the same state at the point of target processing as it would be in a neutral trial. To measure the distracting effect of the nontarget in the cued color, it makes sense to use only the invalid trials in which the nontarget in the cued color is presented before the target. Therefore, we reanalyzed the invalid-trial data, taking into account the relative positions of the cuedcolor distractor and the target. When the nontarget in the cued color appeared one to three frames before the target frame, performance was significantly better than when it appeared earlier than that $[F(1,23)=4.41, p<.05]$.

This finding is in contradiction with a result obtained by Folk et al. (2002). In their RSVP paradigm, distractor items presented in the same color as the one defining the target impaired performance when presented one or two frames before the target. The discrepancy between these results and ours might be caused by repetitions of the cued color, which were present in the experiment of Folk et al., but not in ours. Fox and de Fockert (2001) found that repetition of color could lead to interference similar to the effect of inhibition of return (IOR) observed for repetition of location (Posner \& Cohen, 1984). Since in our experiment the cued color was displayed only once, no inhibition by repetition of color could occur. Instead, the appearance of a letter in the cued color alerted the participants and caused them to process the items that followed immediately more fully.

If the cued color appeared too early, however, processing of the items following the cued color might still 
have been in progress when the target actually appeared, so performance dropped. A comparison with trials in which the color cue was more than three items before the target frame with neutral trials showed a highly significant 5.3\% advantage for neutral trials over invalid trials $[F(1,23)=9.08, p<.01]$ and confirms that an invalid cue leads to processing costs.

In the preceding analysis, accuracy was examined as a function of the sequential position of the target relative to the nontarget in the cued color, but this analysis could be criticized because it does not take the absolute position of the target into account. Targets appearing several positions before the cued nontarget are more likely to appear early in the 15-item sequence, for example, and those appearing several positions after the cued nontarget are more likely to appear late in this sequence. Thus, it is possible that the effects of relative position described above are actually mediated by absolute position, with which they are confounded. To eliminate this confounding, we carried out a further analysis in which we calculated a separate neutral comparison value for each relative position. For each invalid trial, we noted not only its actual accuracy, but also the accuracy of neutral trials with the target in the same absolute position. The latter neutral-trial accuracies were then averaged across trials as a function of the relative position of the target, yielding a separate neutral comparison score for each relative position. Figure 1 displays the results of this analysis, and the resulting pattern confirms the conclusions from the previous analysis. When the cued nontarget was presented within three frames before the target, performance was better for invalid trials than for neutral trials. When the cued nontarget was presented earlier than that, the participants were, on average, $4.73 \%$ less accurate for invalid trials than for neutral trials.

As in Experiment 1, RT differed for the upper- and the lowercase versions of the target $[F(1,22)=26.41, p<$ $.001]$, with faster responses for the uppercase targets. Also, as in Experiment 1, the effect of case on RT decreased at later target positions $[F(4,88)=4.59, p<$ .01]. As before, accuracy was not significantly affected by the case of the target, but the interaction between case and validity was significant $[F(2,44)=7.72, p<.005]$. The differences between upper- and lowercase targets were small for valid and invalid trials $(0.1 \%$ and $2.2 \%$, respectively), with responses for uppercase targets being more accurate. Again, the main difference was caused by neutral trials, for which the difference was $9.8 \%$, with responses for lowercase targets being more accurate. A further analysis again showed a response bias, with the participants giving lowercase responses more frequently than uppercase responses $(54.9 \%$ vs. $45.1 \%)$ for this type of trial. In the neutral condition, guesses might have been more common, because the absence of the cued color did not lead the participants to attend to one particular item in the sequence. In the invalid condition, on the other hand, the increased alertness caused by the cued nontarget apparently led to a benefit in perfor-

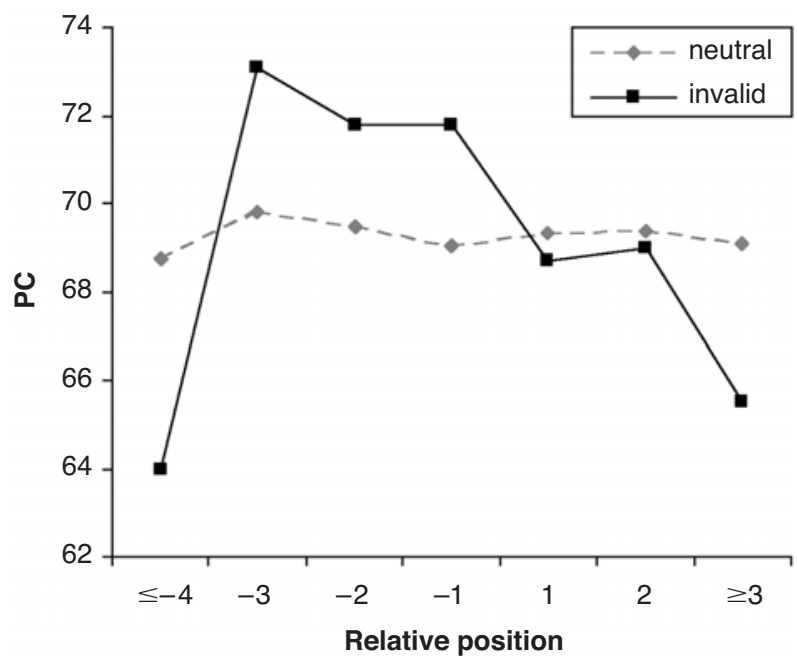

Figure 1. Accuracy of responses in invalid trials as a function of the position of the target relative to the position of the nontarget appearing in the cued color. For comparison, the accuracy of the neutral condition has been adjusted to match the absolute positions within the sequence of the targets in the invalid trials. PC, percentage correct.

mance whenever the real target followed within three frames of the cued nontarget, making guesses necessary less often in invalid trials than in neutral ones.

In addition, the interaction of target case and target position was significant in the analysis of accuracy $[F(4,88)=$ $3.66, p<.01]$. For the first 12 target positions of the sequence, differences in accuracy between upper- and lowercase targets were small-ranging from $-2.1 \%$ to $+2.1 \%$. In contrast, when the target was in Positions 13-15, the difference was $8.8 \%$. This increased difference probably resulted from response bias, because lowercase responses were more frequent $(54.4 \%)$ than uppercase responses (45.6\%). It might be that after 10 letters without the target, the participants thought that they had missed it and, therefore, prepared for a guess in the direction of the case that was more likely to be missed - namely, lowercase. As in Experiment 1, this interaction was further modified by group, with responses to lowercase targets executed with the right hand increasing in accuracy much more than responses for uppercase targets when the target was presented in the last 3 positions of the sequence $[F(4,88)=2.61, p<.05]$. It seems that the participants not only chose to respond more often with the lowercase response key, but also chose to respond more often with their dominant hand.

Further analyses were conducted for error trials in the invalid condition. For these trials, the responses given by the participants were compared with respect to the case of the distractor letter presented in the cued color. Responses were consistent with the case of this distractor letter in $55.7 \%$ of these trials, and this value was significantly greater than chance $[F(1,23)=7.17, p<.02]$. This finding supports the idea that the effect of color 
cuing arises because participants select the letter in the cued color for further processing (i.e., perceptual identification), rather than because they search for a template with a certain color/target conjunction.

In summary, the results of this experiment support the conclusion of the first experiment that color can be used for direct selection of items for further processing. As in the first experiment, the validity of the cue had a significant effect on performance. In addition, the results suggest that this difference was due both to beneficial effects of the valid color cue and to costs associated with the appearance of a distractor letter in the cued color. The size of the facilitory effect of color cuing in valid trials, as compared with neutral trials, was $7.6 \%$. When the neutral trials are compared with the invalid trials in which the misleading color was presented before the target letter, the cost of invalid color cuing was $5.3 \%$ when the cued color was presented more than three frames earlier than the target frame. Furthermore, in invalid trials, the participants responded more often to the case of the cued-color distractor letter than was expected by chance. This suggests that the participants searched initially for the cued color and sometimes reacted to the case of this cued letter even though it was not the target letter. This supports the hypothesis that color served as a selector, rather than being part of a search for a combined color and shape template.

\section{GENERAL DISCUSSION}

In two experiments, we examined whether the usefulness of color for perceptual selection is mediated entirely by location. We provided color cues in an RSVP paradigm without variation in location by using frames with single stimulus letters at fixation. The participants had to discriminate upper- from lowercase target letters within each sequence of colored letters. Performance was better when the target appeared in the cued color than when it did not. This suggests that advance color information allows direct selection of objects for further processing and that such selection is not completely mediated by location.

It also seems that the observed direct selection by color mainly influenced perceptual encoding. RSVP tasks have been shown to be useful for exploring the integration of information to a percept (Botella \& Eriksen, 1992; McLean et al., 1983). The use of an RSVP paradigm and a task that requires discrimination between upper- and lowercase versions of letters should focus in on perceptual processing, rather than on postidentification processes. In addition, the finding that color information affects accuracy suggests an influence early in information processing. An influence on later stages of processing (e.g., at the level of the response) would have led to an advantage for RT only.

In the two experiments described here, an RSVP paradigm was used. A special feature of these tasks is that selection takes place in time, rather than in space. In spa- tial search tasks, color has been shown to be useful as a guide to target location (e.g., Brawn \& Snowden, 1999). It can be argued that a function of color cues in the time domain is analogous to its guidance function in the space domain. According to that view, color cues aided performance by enabling the participants to select the target frame more efficiently. This is likely to be the cause of some, but not all, of the benefit associated with valid color cuing. We think it does not account for all of the benefit, because selection of a target frame by color is actually not that good. Many errors are observed in experiments in which participants are asked to search for a target letter defined by a specified color (e.g., Botella \& Eriksen, 1992; McLean et al., 1983). These experiments had a similar number of frames and a similar presentation rate, as compared with the two experiments reported here. Given that selection of a given frame by color is fairly ineffective, it seems likely that the advantage observed here is at most partly due to more efficient selection in time and at least partly due to direct selection by color.

The present findings of benefits with color cuing in an RSVP paradigm are in contradiction to several other results, reviewed in the introduction, suggesting that the only selective function of color is to guide spatial attention to a specific location on which attention should be focused (e.g., Brawn \& Snowden, 1999; Moore \& Egeth, 1998; Põder, 2001; Shih \& Sperling, 1996; Tsal \& Lavie, 1993). In some of these studies, however, location was also varied in the experimental design (Shih \& Sperling, 1996; Tsal \& Lavie, 1993), in contrast to our experiments, in which stimuli were superimposed so that location did not vary. This difference in design might be at least partly responsible for the contradictory results. For example, it is possible that location is dominant for selection processes whenever it varies and that selective qualities of other features are overshadowed by the dominance of location. In that case, the selective qualities of other features could emerge only when location is not allowed to vary. This view is quite compatible with the claim that location has a special status in selective attention (Nissen, 1985; Tsal \& Lamy, 2000). Yet, on this view, location is not special in the sense that it is the only attribute that enables selection for further processing but, rather, is special in that it dominates other features during selection.

The above location dominance account suggesting that color is not useful for selection - at least beyond what is mediated by location - can explain the difference between our results and previous results whenever location varies. But it cannot explain the differences between our results and those in Põder (2001). Põder also used an RSVP task with presentation of only one stimulus at a time, so location was not varied in his study either. Instead, the reason that Põder found no advantage of color cuing may have been that he included many items in the cued color (cf. Shih \& Sperling, 1996). As was discussed in the introduction, in this experiment, the color cue re- 
duced the number of to-be-attended stimuli only in half, whereas in our experiment the cue reduced the number of such stimuli to only one.

There are at least two reasons why the cuing effect would be expected to be largest when it enables selection of a single stimulus and why it would diminish when it causes selection of a larger number of attended stimuli. One reason is based on the idea that selected items share limited capacity. When $N$ items are presented, suppose that each is processed with capacity $1 / N$ in the absence of any cue. When $M<N$ items are cued, each of the cued items is processed at the higher capacity level of $1 / M$. The greatest cuing benefit clearly arises when $M=1$, because in this case all processing capacity can be devoted to a single item. The second reason is strategic: If it is effortful to use the cue, participants may be more likely to engage in this effort when the payoff is larger - that is, when it allows a greater increase in the focus of processing capacity on the target.

We suspect that the main reason color cuing was more effective in our experiments than in previous ones has to do with the fact that we used color to cue a single item, and in fact, data from experiments with location cues support this position. For one thing, in most experiments showing that location cues could be used successfully, this cue normally reduced the possible target locations in the display to a single likely target location (e.g., Posner et al., 1980; Treisman, 1985). Moreover, in studies in which location cues were used to select out more than one location, cuing effects generally decreased rapidly as more locations were cued. For example, Palmer, Ames, and Lindsey (1993) cued two, four, or eight locations within a display of 8 stimuli. Performance was better when two locations were cued, rather than four, but there was no difference between four and eight, indicating a limit to the effectiveness of multiple location cues. Similarly, Kröse and Julesz (1989) examined the effectiveness of multiple location cues within a circular display of 12 stimuli. Cuing was effective for up to three locations but was not effective when five locations were cued. Thus, location cuing seems to be beneficial only if just a few locations are cued. It is reasonable to assume that an analogous limitation would be present with color cuing. In general, it might be that cuing information is used only when it reduces the to-be-attended stimulus set by a significant amount, which might be reflected in a critical proportion of the total stimuli in the display. This proportion probably varies across tasks, however. Brawn and Snowden (1999) found a benefit in color cuing when $50 \%$ of the display stimuli had one color, whereas Põder (2001) did not find any effect of color information, although his cue also led to a reduction of the stimulus set by half.

Finally, certain properties of the stimulus set might also have contributed to our finding of an advantage for color cues not found by previous researchers. According to the attentional engagement theory (Duncan \& Humphreys, 1989), similarity and dissimilarity of targets and nontar- gets heavily influences performance. The more dissimilar a target is from all the nontargets, the easier is the selection. It might be that colors were easier to distinguish from each other than were the letters, increasing their usefulness as cues. If the participants found the colors much easier to distinguish than the letters, they would presumably have been more inclined to use the color cues.

To investigate the relative color/letter discriminabilities in our stimulus set, we conducted a further pilot study that matched the conditions of the original experiments as closely as possible. In this study, 12 participants were presented with the same colored letter RSVP sequences as those used in the main experiments. They performed yes/no detection tasks, and in alternating blocks, the target was either (1) a prespecified color or (2) a prespecified letter, which could be presented in its upper- or lowercase form. For the color detection task, a new target color was provided on a trial-to-trial basis, and for the letter detection task, target letters changed on a block basis, as in the main experiments. The accuracy in the color detection task was significantly better than that in the letter detection task $[F(1,10)=15.15, p<$ $.005]$, indicating that colors were indeed easier to distinguish from each other than letters were. This difference in discriminability might have contributed to the cuing effect found.

In our second experiment, we examined whether the advantage found for the valid condition was caused by a benefit for the valid trials, a cost for the invalid trials, or both. Following the recommendations of Jonides and Mack (1984), the cues and the trials were exactly the same in all three conditions, except that the cues would be expected to facilitate processing in the valid condition and to interfere with it in the invalid condition. It is, as usual, arguable whether the neutral condition represents an absolutely neutral condition, but it seems likely that this condition would evoke very little facilitation or inhibition of task processing, relative to the other two conditions with which it was compared. The comparison among the valid, neutral, and invalid conditions revealed that color cuing had both facilitory and inhibitory effects on performance. The participants were able to process a letter in the expected color especially efficiently but were disadvantaged by misleading color information.

There are several possible mechanisms by which advance color information could improve performance in the RSVP task. First, participants might perform the task by creating templates of two compound stimuli (e.g., a red $\mathrm{H}$ and $\mathrm{a}$ red $\mathrm{h}$ ) and searching for these templates within the RSVP sequence. If they did that, the participants would focus only on the two conjunction (i.e., color and shape) templates and not on the single features of the compound stimuli. In that case, however, the cued color should have no influence on the responses in invalid trials, because in those trials the target templates never occurred. But our data show that in these trials, the misleading color cue led the participants to respond more often than chance to the case in which the nontarget let- 
ter appeared in the cued color. This suggests that the participants used color information separately from shape information and did not rely exclusively on a conjunction of the two features.

Two other possible mechanisms for the advantage of color cues in our task are signal enhancement and noise reduction (Shiu \& Pashler, 1994). Cheal and Gregory (1997) found evidence for both signal enhancement and noise reduction in a spatial-cuing task, but the present data do not allow us to evaluate the possible contributions of these mechanisms in the present RSVP task. The idea of signal enhancement could certainly explain our results. Specifically, more processing capacity might be allocated to the item of the cued color as an effect of the cuing, and this extra capacity could enhance shape perception for that item. In valid trials, the processing of target shape would be enhanced, improving discrimination. In neutral trials, no letter would be singled out, so all the letters would be processed equally - that is, target processing would not be enhanced. In invalid trials, the wrongly cued nontarget would receive more processing capacity, leading to some reduction in the capacity available for processing the target, although the appearance of the cued color could have an alerting effect for the following three items. As was discussed in the introduction, the idea of signal enhancement by color cuing was investigated and rejected by Moore and Egeth (1998), but their conclusions may not be applicable to the present paradigm for a number of reasons. Most important, their displays included variation in location, as well as in color, so location may have dominated. In addition, their color cues allowed selection of only half of the display items - not a single item-so the participants may not have been sufficiently motivated to use them.

Another possible mechanism is noise reduction. With this mechanism, cuing benefits are gained by eliminating potentially confusing nontarget information from the decision process (Shiu \& Pashler, 1994). Because the cued color appeared only once in each of the present RSVP sequences, many nontargets could have been excluded. Thus, the idea of noise reduction could also easily explain the advantage for the valid condition, because only in this condition could all of the nontargets be excluded from the decision. The cost of misleading cuesthat is, the disadvantage of the invalid condition relative to the neutral condition-is more difficult to explain with this account, however. On a strict interpretation of the noise reduction mechanism, in neutral and invalid trials, the target letter would always be excluded from the decision process because it did not appear in the cued color. This would seem to predict equal and chance performance in these two conditions, but actual performance was different in these two conditions and was well above the chance level. It would still be possible to account for the results, however, in terms of a graded noise reduction mechanism in which the letters in uncued colors did contribute - albeit more weakly than the letters in the cued color - to the decision process.

\section{REFERENCES}

Botella, J., \& ERIKSEN, C. W. (1992). Filtering versus parallel processing in RSVP tasks. Perception \& Psychophysics, 51, 334-343.

Brawn, P., \& SnOwden, R. J. (1999). Can one pay attention to a particular color? Perception \& Psychophysics, 61, 860-873.

CARTER, R. C. (1982). Visual search with color. Journal of Experimental Psychology: Human Perception \& Performance, 8, 127-136.

CAVE, K. R., \& PASHLER, H. (1995). Visual selection mediated by location: Selecting successive visual objects. Perception \& Psychophysics, 57, 421-432.

Cheal, M., \& Gregory, M. (1997). Evidence of limited capacity and noise reduction with single-element displays in the location-cuing paradigm. Journal of Experimental Psychology: Human Perception \& Performance, 23, 51-71.

Chun, M. M. (1997). Temporal binding errors are redistributed by the attentional blink. Perception \& Psychophysics, 59, 1191-1199.

Duncan, J., \& Humphreys, G. W. (1989). Visual search and stimulus similarity. Psychological Review, 96, 433-458.

Egeth, H. E., Virzi, R. A., \& Garbart, H. (1984). Searching for conjunctively defined targets. Journal of Experimental Psychology: Human Perception \& Performance, 10, 32-39.

Folk, C. L., Leber, A. B., \& Egeth, H. E. (2002). Made you blink! Contingent attentional capture produces a spatial blink. Perception \& Psychophysics, 64, 741-753.

FOX, E., \& DE FOCKERT, J.-W. (2001). Inhibitory effects of repeating color and shape: Inhibition of return or repetition blindness? Journal of Experimental Psychology: Human Perception \& Performance, 27, 798-812.

GARNER, W. R. (1987). Location and color as cuing dimensions in contingent classification. Perception \& Psychophysics, 41, 202-210.

Gold, J. M., \& PRATT, J. (2001). Is position "special" in visual attention? Evidence that top-down processes guide visual selection. Canadian Journal of Experimental Psychology, 55, 261-270.

Goldstein, B. E., \& FInK, S. I. (1981). Selective attention in vision: Recognition memory for superimposed line drawings. Journal of Experimental Psychology: Human Perception \& Performance, 7, 954967.

Green, B. F., \& Anderson, L. K. (1956). Color coding in a visual search task. Journal of Experimental Psychology, 51, 19-24.

Humphreys, G. W. (1981). Flexibility of attention between stimulus dimensions. Perception \& Psychophysics, 30, 291-302.

Huynh, H. (1978). Some approximate tests for repeated measurement designs. Psychometrika, 43, 161-175.

Jonides, J., \& MACK, R. (1984). On the cost and benefit of cost and benefit. Psychological Bulletin, 96, 29-44.

Kaptein, N. A., Theeuwes, J., \& van der Heijden, A. H. C. (1995). Search for a conjunctively defined target can be selectively limited to a color-defined subset of elements. Journal of Experimental Psychology: Human Perception \& Performance, 21, 1053-1069.

KIM, M.-S., \& CAVE, K. R. (2001). Perceptual grouping via spatial selection in a focused-attention task. Vision Research, 41, 611-624.

Kröse, B. J. A., \& Julesz, B. (1989). The control and speed of shifts of attention. Vision Research, 29, 1607-1619.

LaBerge, D., \& Brownston, L. S. (1974). Control of visual processing by color cueing. Bulletin of the Psychonomic Society, 4(4B), 417418.

LAMbert, A. J., \& Corban, R. (1992). Spatial attention and expectancy for colour, category and location: Further evidence against the spotlight model. Acta Psychologica, 81, 39-51.

LAmY, D., \& TsaL, Y. (2000). Object features, object locations, and object files: Which does selective attention activate and when? Journal of Experimental Psychology: Human Perception \& Performance, 26, 1387-1400.

Mclean, J. P., Broadbent, D. E., \& Broadbent M. H. P. (1983). Combining attributes in rapid serial visual presentation tasks. Quarterly Journal of Experimental Psychology, 35A, 171-186.

Miller, J., \& SCHRöTER, H. (2002). Online response preparation in a rapid serial visual search task. Journal of Experimental Psychology: Human Perception \& Performance, 28, 1364-1390.

Moore, C. M., \& Egeth, H. (1998). How does feature-based attention 
affect visual processing? Journal of Experimental Psychology: Human Perception \& Performance, 24, 1296-1310.

NisSEN, M. J. (1985). Accessing features and objects: Is location special? In M. I. Posner \& O. S. M. Marin (Eds.), Attention and performance XI (pp. 205-219). Hillsdale, NJ: Erlbaum.

Palmer, J., Ames, C. T., \& Lindsey, D. T. (1993). Measuring the effect of attention on simple visual search. Journal of Experimental Psychology: Human Perception \& Performance, 19, 108-130.

PõDER, E. (2001). Size-based selection in rapid serial visual presentation. Vision Research, 41, 2221-2234.

PosNer, M. I., \& CoHEN, Y. (1984). Components of visual orienting. In H. Bouma \& D. G. Bouwhuis (Eds.), Attention and performance X: Control of language processes (pp. 531-556). Hillsdale, NJ: Erlbaum.

Posner, M. I., SNyder, C. R. R., \& Davidson, B. J. (1980). Attention and the detection of signals. Journal of Experimental Psychology: General, 109, 160-174.

Rock, I., \& Gutman, D. (1981). The effects of inattention on form perception. Journal of Experimental Psychology: Human Perception \& Performance, 7, 275-285.

SHIH, S.-I, \& SPERLING, G. (1996). Is there feature-based attentional selection in visual search? Journal of Experimental Psychology: Human Perception \& Performance, 22, 758-779.

SHIU, L., \& PASHLER, H. (1994). Negligible effects of spatial precuing on identification of single digits. Journal of Experimental Psychology: Human Perception \& Performance, 20, 1037-1054.

SPERLING, G. (1960). The information available in brief visual presentations. Psychological Monographs, 74, 1-29.

Theeuwes, J. (1989). Effects of location and form cuing on the allocation of attention in the visual field. Acta Psychologica, 72, 177-192.

Tipper, S. P., \& Cranston, M. (1985). Selective attention and priming: Inhibitory and facilitatory effects of ignored primes. Quarterly Journal of Experimental Psychology, 37A, 591-611.

Treisman, A. M. (1985). Preattentive processing in vision. Computer Vision, Graphics, \& Image Processing, 31, 156-177.
Tsal, Y., \& Lamy, D. (2000). Attending to an object's color entails attending to its location: Support for location-special views of visual attention. Perception \& Psychophysics, 62, 960-968.

Tsal, Y., \& LaVIE, N. (1988). Attending to color and shape: The special role of location in selective visual processing. Perception \& Psychophysics, 44, 15-21.

TSAL, Y., \& LAVIE, N. (1993). Location dominance in attending to color and shape. Journal of Experimental Psychology: Human Perception \& Performance, 19, 131-139.

van der Heidden, A. H. C., Kurvink, A. G., De Lange, L., De Leeuw, F., \& VAN DER GEEST, J. N. (1996). Attending to color with proper fixation. Perception \& Psychophysics, 58, 1224-1237.

VON WRIGHT, J. M. (1968). Selection in visual immediate memory. Quarterly Journal of Experimental Psychology, 20, 62-68.

\section{NOTE}

1. This conclusion also receives strong support from studies in which color formed part of the target description, rather than simply being a potentially informative cue. Using the partial report paradigm of Sperling (1960), for example, von Wright (1968) found that color could be used quite successfully to guide the report of letters from a briefly presented letter array. Similarly, in an RT study by Egeth, Virzi, and Garbart (1984), participants had to search for a red O in a multiple-item display. The authors varied the total display size, while keeping the number of red letters constant. An increase of total display size had no effect on the search times for the target letter, indicating that the participants were able to limit their search to red items. Later, these findings were extended by Kaptein, Theeuwes, and van der Heijden (1995), who showed that within constant total display sizes, search times vary with the number of items sharing the target color.

(Manuscript received October 21, 2003; revision accepted for publication July 7, 2004.) 